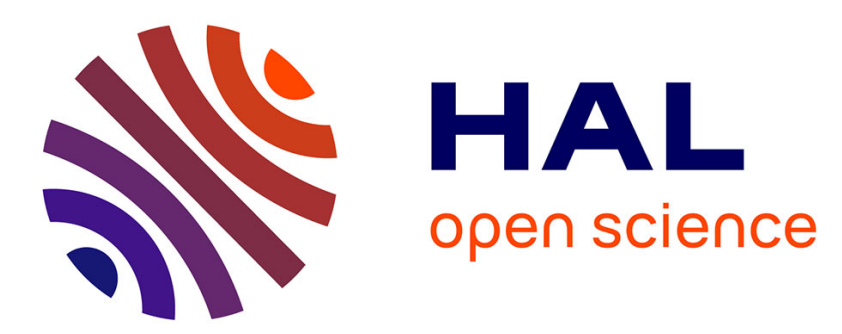

\title{
Experimental characterization of the coupled conductive and radiative heat transfer in ceramic foams with a flash method at high temperature
}

\author{
Morgan Sans, Vincent Schick, Gilles Parent, Olivier Farges
}

\section{To cite this version:}

Morgan Sans, Vincent Schick, Gilles Parent, Olivier Farges. Experimental characterization of the coupled conductive and radiative heat transfer in ceramic foams with a flash method at high temperature. International Journal of Heat and Mass Transfer, 2020, 148, pp.119077. 10.1016/j.ijheatmasstransfer.2019.119077 . hal-02410598

\author{
HAL Id: hal-02410598 \\ https://hal.science/hal-02410598
}

Submitted on 15 Dec 2019

HAL is a multi-disciplinary open access archive for the deposit and dissemination of scientific research documents, whether they are published or not. The documents may come from teaching and research institutions in France or abroad, or from public or private research centers.
L'archive ouverte pluridisciplinaire HAL, est destinée au dépôt et à la diffusion de documents scientifiques de niveau recherche, publiés ou non, émanant des établissements d'enseignement et de recherche français ou étrangers, des laboratoires publics ou privés. 


\title{
Experimental characterization of the coupled conductive and radiative heat transfer in ceramic foams with a flash method at high temperature
}

\author{
Morgan Sans, Vincent Schick, Gilles Parent, Pr*, Olivier Farges \\ Laboratoire Energies et Mécanique Théorique et Appliquée (LEMTA), Universitéde Lorraine - CNRS, UMR 7563, Vandoeuvre-lés-Nancy 54505, France
}

A R T I C L E I N F O

Keywords:

Monte-Carlo method

Heat conduction

Thermal radiation

Flash method measurement

Equivalent thermal conductivity

Ceramic foams

Equivalent optical thickness

\begin{abstract}
A B S T R A C T
During recent decades, numerous studies on ceramic foams improved their efficiency and made their use possible in a myriad of engineering applications. Some studies showed that a good understanding of the link between structural parameters (porosity, pore diameter, or tortuosity) and thermal properties could lead to a better system performance. Ceramic foams are often used at high temperatures, where radiation plays an important role. This paper explores, both conductive and radiative heat transfers in such media, using a flash method experiment. The direct model developed is based on the resolution of the Energy Balance Equation (EBE), solved by a Finite Volume Method and the Radiative Transfer Equation (RTE), computed with an Optimized Emission Reciprocity Method (OERM). An inversion procedure made it possible to simultaneously characterize both equivalent conductivity for conduction and equivalent optical thickness for radiation of structured and random foams with different pore size distributions at $800{ }^{\circ} \mathrm{C}$. Results showed that the influence of each heat transfer mode can be characterized separately with single experiment and a good agreement was found between the developed model and the experimental measurements. Radiative transfers can be described by a single equivalent, Beerian and gray extinction coefficient dependings strongly on the size of the cells and the organization of the solid matrix. The equivalent conductivity depends on the porosity, the ceramic constituting the solid matrix, the organization of the porous medium and the internal porosity. We estimated the associated equivalent total and radiative conductivities, allowing the quantification of the conduction/radiation ratio, for a wide variety of structures.
\end{abstract}

\section{Introduction}

Due to their numerous interesting thermal, mechanical and optical properties, open-cell foams are commonly used in different engineering applications [1]. A wide diversity of materials, geometries and structural parameters makes it possible to cover many processes, such as porous burners [2,3], insulators [4,5], heat exchangers [6-8], volumetric solar absorbers [9,10], catalysis $[11,12]$ or gas-phase heat recovery [13].

Many coupled physicals phenomena, such as conduction, convection, transport, radiation or chemical reaction depend on the geometry of the foam, which can play an important role in the efficiency of the system. For example, Chavez et al. [14] showed that higher porosities lead to a higher temperature and thus better energy conversion, while [15] demonstrated that low cell sizes

\footnotetext{
* Corresponding author.

E-mail address: gilles.parent@univ-lorraine.fr (G. Parent).
}

improve convective exchanges within the foam. In order to design and study a system that includes a porous medium with a complex geometry, a numerical solver can be used. However, the computation time needed to obtain a precise solution can be lengthy. Nevertheless, thise problem can be simplified by considering foam as a homogeneous medium with equivalent properties.

Equivalent Thermal Conductivity (ETC) represents the overall thermal diffusion through the media, which includes conduction and radiation in the solid phase and conduction, convection and radiation in the fluid phase. ETC estimation was widely studied in the literature [16]. Models developed provide analytical solutions, essentially based on the structural parameters, empirical analysis and numerical computations.

Theoretically, in the simplest case, assuming that the fluid and solid phase are parallel or perpendicular to the heat flux, two asymptotic models are written as:

$\lambda_{\perp}=\epsilon \cdot \lambda_{\mathrm{f}}+(1-\epsilon) \cdot \lambda_{\mathrm{s}} \frac{1}{\lambda_{/ /}}=\frac{\epsilon}{\lambda_{\mathrm{f}}}+(1-\epsilon) \frac{1}{\lambda_{\mathrm{s}}}$ 


\begin{tabular}{|c|c|}
\hline \multicolumn{2}{|c|}{ Nomenclature } \\
\hline \multicolumn{2}{|c|}{ Latin Symbols } \\
\hline$\Delta R$ & distance traveled through an element(m) \\
\hline & specific heat $\left(\mathrm{J} \mathrm{Kg}^{-1} \mathrm{~K}^{-1}\right)$ \\
\hline$K$ & adjustment parameter $(-)$ \\
\hline$P_{\mathrm{q}}$ & power carried by the quantum(W) \\
\hline$S$ & surface $\left(\mathrm{m}^{2}\right)$ \\
\hline$S_{\mathrm{r}}$ & radiative volumic source term $\left(\mathrm{W} \mathrm{m}^{-3}\right)$ \\
\hline$V$ & volume $\left(\mathrm{m}^{-3}\right)$ \\
\hline DL & digital levels $(-)$ \\
\hline$D_{\text {cell }}$ & cell size of the foam (m) \\
\hline$T$ & temperature $(\mathrm{K})$ \\
\hline$t$ & time $(s)$ \\
\hline$\vec{q}$ & heat flux density $\left(\mathrm{W} \mathrm{m}^{-2}\right)$ \\
\hline$I$ & radiant intensity $\left(\mathrm{W} \mathrm{m}{ }^{-2} \mathrm{sr}^{-1} \mathrm{~Hz}^{(-1)}\right)$ \\
\hline$\vec{R}$ & vector coordinate $(\mathrm{m})$ \\
\hline$r$ & radial coordinate $(\mathrm{m})$ \\
\hline$z$ & axial coordinate $(\mathrm{m})$ \\
\hline$\vec{n}$ & unit normal vector $(-)$ \\
\hline$h$ & 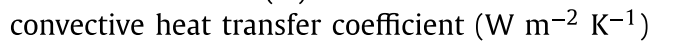 \\
\hline$R$ & radius of the sample $(\mathrm{m})$ \\
\hline$L$ & thickness of the system $(\mathrm{m})$ \\
\hline$\Delta t$ & interval of time $(s)$ \\
\hline$\Delta r$ & $\begin{array}{l}\text { size of the discretised volume along the radial di- } \\
\text { mension }(\mathrm{m})\end{array}$ \\
\hline$\Delta z$ & $\begin{array}{l}\text { size of the discretised volume along the axial di- } \\
\text { mension }(\mathrm{m})\end{array}$ \\
\hline$N$ & number of element \\
\hline$P$ & radiative power $(\mathrm{W})$ \\
\hline$J$ & cost function \\
\hline$N_{r}$ & $\begin{array}{l}\text { number of spatial discretization along the radial } \\
\text { axis }\end{array}$ \\
\hline$N_{z}$ & number of spatial discretization along the axial axis \\
\hline$N_{t}$ & number of time discretization \\
\hline$\underline{\underline{D}}$ & diagonal matrix \\
\hline$\overline{\bar{S}}$ & sensitivity \\
\hline $\bar{T}$ & space averaged temperature $(\mathrm{K})$ \\
\hline$a$ & thermal diffusivity $\left(\mathrm{m}^{2} \mathrm{~s}^{-1}\right)$ \\
\hline$e$ & thickness of the soleplate (m) \\
\hline$e_{\text {foam }}$ & thickness of the foam sample (m) \\
\hline \multicolumn{2}{|c|}{ Greek Symbols } \\
\hline$\alpha$ & structural parameter of the inversion \\
\hline & thermal conductivity $\left(\mathrm{W} \mathrm{m} \mathrm{m}^{-1} \mathrm{~K}^{-1}\right)$ \\
\hline$\sigma_{\mathrm{b}}$ & standard deviation of the noise \\
\hline$\rho$ & density $\left(\mathrm{Kg} \mathrm{m}^{-3}\right)$ \\
\hline$\vec{\Omega}$ & direction $(-)$ \\
\hline$v$ & frequency $(\mathrm{Hz})$ \\
\hline$\kappa_{a}$ & absorption coefficient $\left(\mathrm{m}^{-1}\right)$ \\
\hline$\kappa_{d}$ & scattering coefficient $\left(\mathrm{m}^{-1}\right)$ \\
\hline$\beta$ & extinction coefficient $\left(\mathrm{m}^{-1}\right)$ \\
\hline$\Omega$ & solid angle (sr) \\
\hline$\phi$ & excitation heating power $\left(\mathrm{W} \mathrm{m}^{-2}\right)$ \\
\hline$\sigma$ & Stefan-Boltzmann constant $\left(\mathrm{W} \mathrm{m}^{-2} \mathrm{~K}^{-4}\right)$ \\
\hline$\varepsilon$ & emissivity (-) \\
\hline$\Gamma$ & domain \\
\hline$\partial \Gamma$ & contour \\
\hline$\Phi$ & scattering phase function $(-)$ \\
\hline$\mu$ & relaxation coefficient $(-)$ \\
\hline$\epsilon$ & foam porosity $(-)$ \\
\hline$\theta$ & normalized temperature $(-)$ \\
\hline$\tau$ & optical thickness $\left(\tau=\beta \cdot e_{\text {foam }}\right)(-)$ \\
\hline
\end{tabular}

\begin{tabular}{|ll|}
\hline \multicolumn{3}{|c|}{ Subscripts } \\
eff & effective \\
c & conduction \\
r & radiation \\
$v$ & spectral \\
s & solid \\
wa & wall \\
f & fluid \\
sp & specular \\
ref & reference \\
ext & external part of the sample \\
t & total \\
tot & equivalent total (conduction and radiation) property \\
foam & equivalent property of the foam \\
sole & soleplate \\
$\infty$ & environment \\
$i$ & computed volume or surface elements \\
$j$ & other volume or surface elements of the domain \\
max & maximum \\
opt & optimal \\
min & minimum \\
$n$ & nth parameter of the inversion \\
exp & from experiment \\
mod & from model \\
bulk & struts \\
conv & convection \\
Superscripts \\
bb & blackbody \\
$k$ & at $k$ th iteration in time \\
e & emitted \\
ea & emitted and absorbed \\
$p$ & at pth iteration of the inversion \\
OERM & Optimized Emission Reciprocity Method \\
exch & exchanged \\
$r, z$ & radial and axial \\
\hline
\end{tabular}

On the one hand, Miller [17] proposed an extension of this asymptotic approach in the case of a discontinuous phase. Moreover, Hashin et al. [18] suggested an equivalent mathematical model (i.e., an upper and lower bound model), which is more realistic for an isotropic two-phase material. However, both models assume that one of the dispersed phases is not inter-connected. Landauer's Effective Medium Theory [19], which considers a two-phase randomly distributed situation, proposed a more general formulation. Yet, these models are still not applicable to an open-cell medium.

On the other hand, many authors presented models based on a unit-cell approach. In these models, the structure of the foam is assumed ideal and is described with a unitary pattern, which is repeated infinitely. Hence, slice by slice, the effective thermal resistance of the foam is computed as the sum of all the equivalent thermal resistances. This principle was developed for many different unit-cell geometries. For example, a 3-D cubic cell unit study was proposed by Paek et al. [20], while Hsu et al. investigated a packed bed sphere [21]. Moreover, it was widely demonstrated that an open-cell foam can be represented by a polyhedron, such as a tetrakaidecahedron. In 2-D geometry, Calmidi et al. [22] and Bhattacharya et al. [23] proposed a semi-analytical model with either square nodes or spherical nodes, joined by a solid ligament. Similarly, Boomsma and Poulikakos [24] extended the resolution to three dimensions with cubic nodes, joined by cylindrical struts. This last model was found to be in good agreement with experimental data for high porosity aluminum foams, filled with air or water. Moreover, the analysis demonstrated that cell size has no influence on the ETC. The conductivity of the solid phase and the 
shape of the struts are the two parameters with the most important roles.

Experimental and numerical studies at ambient temperature were then performed to explore the validity of the theoretical models described above. An empirical correlation was suggested by fitting either numerical computation data or experimental data. Singh and Kasana [25] used the data from Boomsma et al. [24] and expressed effective thermal conductivity as a product of serial and parallel conductivities: $\lambda_{\text {eff }}=\lambda_{/ /}^{F} \cdot \lambda_{\perp}^{(1-F)}$. The empirical constant $F$ adjusts the fraction of solid in the direction of the heat flux and depends on the fluid phase. However, it would be relevant to determine the range of applicability and the possible discrepancies. Coquard and Bailly [26] developed a numerical finite volume method, in order to compute the thermal behavior of open or closed 3-D structures from a tomographic image. This study showed that the shape of the cell and the shape of the cross section have no influence on the heat transfer. However, it confirmed the findings of the previous studies suggesting that the ETC is not only dependent on porosity. In view of the above, a very great deal of literature is available on the study of equivalent thermal properties of heterogeneous media. Interested readers can refer to [16] a recent review of the subject, which gives a broad overview of the studied geometries in different scopes of validity.

Applications of ceramic and metal foams often involve high temperatures, at which radiative transfer plays a leading role. The description of radiative transfer in such media has mostly been studied independently of the other physical phenomena. The identification of the equivalent optical properties can be computed by analytical method, projection method or Monte Carlo Method $[27,28]$. Tancrez and Taine [29] proposed the Radiative Distribution Function Identification (RDFI), which allows the determination of the equivalent radiative properties of the medium. For this prupose, rays are drawn randomly into the medium and a mean free path is deduced from it. With the same objective, GuÃ@velou et al. [30] used a Monte Carlo Ray Tracing (MCRT) method to compute the normal spectral emittance of numerically-generated $\alpha$-SiC foams. Loretz et al. [31] proposed a numerical method based on transmittance, absorbance and reflectance measurement to calculate the equivalent radiative properties.

In addition, the characterization of radiative transfers in porous media and the associated modelling are often handled separately from other modes of heat transport. However, the experimental methods currently used for high temperature characterization on these media do not allow the separation of conduction transfers from radiation transfers. This is due to the stationary aspect of these methods such as the hot guarded plate or hot plane. Only an equivalent total conductivity could be estimated. The estimation of the influence of each transfer mode requires the use of an approximation such as Rosseland's. As highlighted by Mendes et al. [32], such an approximation is not always valid and a significant bias can be observed. These authors identified an equivalent extinction coefficient using a ray-tracing method and showed that the radiative conductivity according to Rosseland overestimates the experimentally measured radiative conductivity. This type of observation was also observed by the same authors by applying a hot plate method using a complete numerical approach [33]. Hence, as explained by Baillis et al. [34], the experimental characterization of the conductive and radiative properties of solid foams at high temperature remains an unsolved problem and an interesting scientific challenge.

Transient characterization methods such as the flash method appear a potentially interesting way forward thanks to their ability to identify several parameters describing different heat transfer modes. The characterization procedure requires the use of a fast and accurate coupled model. Concerning characterization of semi-transparent media, Lazard et al. [35] first proposed a com- plete procedure to estimate the so-called phononic conductivity of silica glass. Moreover, Schick et al. [36] investigated properties of liquid glass at high temperatures with a 3-D model. Niezgoda et al. [37] applied the method more specifically to the study of a silica aerogel and other thermal insulators. However, the possibility of accurately identifying conductive and radiative transfers depends on the experimental and on the different parameters of the problem. These latest studies have shown that the identification of radiative properties was difficult due to a lack of sensitivity or to correlations between parameters. However, for the first time, Coquard et al. [38] characterized both equivalent conductivity and optical thickness simultaneously using a flash method. This work investigated metallic/ceramic foams ( $\mathrm{NiCrAl}$, Fecralloy, Mullite and Zirconia) with small cell sizes $(<1.4 \mathrm{~mm}$ ) over a temperature range of $296 \mathrm{~K}-673 \mathrm{~K}$.

In the present paper, we applied a flash method for different ceramic foams up to $1073 \mathrm{~K}$. A wide variety of geometries (structured and random) or structural parameters (cell size, porosity or organization of the solid matrix) were investigated. Moreover, a direct model, which solves Radiative Transfer Equation (RTE) using an Optimized Emission Reciprocity Method (OERM) was developed and allowed a fast and accurate calculation of the solution.

\section{Methods}

We assumed that the thermal and optical properties of the foam can be homogenized. Hence, the foam is comparable to a semi-transparent medium characterized by an equivalent conductivity and an equivalent extinction coefficient. The characterization procedure and the experimental set up were based on the flash method. The studied foam was placed between two opaque soleplates inside a furnace at a given temperature. $\mathrm{A} \mathrm{CO}_{2}$ laser provided a heat excitation on the front face of the sample, while the temporal evolution of the temperature on the rear face (thermogram) was measured with an infrared camera. The inversion procedure estimates the set of parameters which minimizes the discrepancy between the model and the experiment.

The next part of this paper details, governing equations and boundary conditions. We then explain the numerical model that allows the physical problems to be solved. Finally, the principle of the inversion technique and identifiability of parameters are presented.

\subsection{Governing equations}

Their good mechanical, thermal and optical properties of ceramic foams mean that they are often used for high temperature applications ( $800 \mathrm{~K}$ to $1300 \mathrm{~K}$ ). At such high temperatures, radiation becomes the dominant heat transfer mode. Thus, we consider that a foam is a participating medium where absorbing, emitting and possibly scattering phenomena occur. During a flash method experiment, only a very low increase of the equilibrium temperature $T_{\infty}$ on the rear face ( $1 \mathrm{~K}$ to $2 \mathrm{~K}$ ) is necessary. We can therefore assume that there is no thermal dependence of the parameters in this small temperature range. The first soleplate, which is opaque and grey, absorbs the excitation and homogenizes the heat flux. The second one, made of the same medium, makes it possible to measure the space averaged temperature of the rear face. Heat conduction through the soleplates is described by the Energy Balance Equation (EBE), with the assumption that their properties are homogeneous and isotropic:

$(\rho C p)_{\text {sole }} \frac{\partial T}{\partial t}=-\vec{\nabla} \cdot \overrightarrow{q_{\mathrm{c}}}$ 
The conductive flux is related to the temperature according to the well-known Fourier's law:

$\overrightarrow{q_{\mathrm{c}}}=-\lambda \vec{\nabla} T$

In order to describe a participating medium, the radiative transfer is taken into account through the divergence of the radiative flux $\vec{q}_{\mathrm{r}}$. In this case, the EBE becomes:

$(\rho C p)_{\text {foam }} \frac{\partial T}{\partial t}=-\vec{\nabla} \cdot\left(\overrightarrow{q_{\mathrm{c}}}+\overrightarrow{q_{\mathrm{r}}}\right)=-\vec{\nabla} \cdot \overrightarrow{q_{\mathrm{c}}}+S_{\mathrm{r}}$

where $S_{\mathrm{r}}=-\vec{\nabla} \cdot \overrightarrow{q_{\mathrm{r}}}$ is the radiative volumetric source term. Let us note that no heat flux through air, due to convection or conduction, is considered because the experiment is performed in a vacuum.

The divergence of the radiative flux term is equivalent to an internal source, which quantifies gain/loss of energy due to radiative exchanges. According to the vector coordinate $\vec{R}$, the radiative heat flux corresponds to the integration of the intensity $I_{v}(\vec{R}, \vec{\Omega})$ over all wavelengths and directions:

$\overrightarrow{q_{\mathrm{r}}}(\vec{R})=\int_{\nu} \int_{\Omega=4 \pi} I_{\nu}(\vec{R}, \vec{\Omega}) \vec{\Omega} \mathrm{d} \Omega \mathrm{d} \nu$

The divergence of $\overrightarrow{q_{\mathrm{r}}}$ is computed as:

$S_{\mathrm{r}}=\vec{\nabla} \cdot \overrightarrow{q_{\mathrm{r}}}=\vec{\nabla} \cdot \int_{v} \int_{\Omega=4 \pi} I_{v}(\vec{R}, \vec{\Omega}) \vec{\Omega} \mathrm{d} \Omega \mathrm{d} v$

$=\int_{\nu} \kappa_{\mathrm{a} \nu}\left(4 \pi I_{\nu}^{\mathrm{bb}}(T)-\int_{\Omega=4 \pi} I_{\nu}(\vec{R}, \vec{\Omega}) \mathrm{d} \Omega\right) \mathrm{d} \nu$

The intensity is determined with the Radiative Transfer Equation (RTE) [39]:

$$
\begin{gathered}
\vec{\Omega} \cdot \vec{\nabla} I_{\nu}(\vec{R}, \vec{\Omega})=-\beta_{v} I_{v}(\vec{R}, \vec{\Omega})+\kappa_{\mathrm{a} v} I_{v}^{\mathrm{bb}}(T(\vec{R}, t)) \\
\quad+\frac{1}{4 \pi} \int_{\Omega^{\prime}=4 \pi} \kappa_{\mathrm{d} \nu} \Phi_{\nu}\left(\overrightarrow{\Omega^{\prime}} \rightarrow \vec{\Omega}\right) I_{v}\left(\vec{R}, \overrightarrow{\Omega^{\prime}}\right) \mathrm{d} \Omega^{\prime}
\end{gathered}
$$

where $\kappa_{\mathrm{d} v}, \kappa_{\mathrm{a} v}$ and $\beta_{v}$ are the spectral scattering, absorption and extinction coefficients, respectively. Because of the high speed interaction of the radiative transfer compared with conductive transfer, intensity is not dependent on time explicitly but implicitly via the temperature field $T(\vec{R}, t)$.

In the case of an opaque wall, the radiative boundary condition is written as:

$$
\begin{aligned}
I_{v}\left(\vec{R}_{\mathrm{wa}}, \vec{\Omega}\right)= & \varepsilon_{v} I_{v}^{\mathrm{bb}}\left(T\left(\vec{R}_{\mathrm{wa}}\right)\right)+\rho_{\mathrm{sp}} I_{\nu}\left(\vec{R}_{\mathrm{wa}},{\overrightarrow{\Omega^{\prime \prime}}}^{\prime}\right) \\
& +\frac{\rho_{\mathrm{d}}}{\pi} \int_{\Omega^{\prime} \cdot \vec{n}<0} I_{\nu}\left(\vec{R}_{\mathrm{wa}}, \vec{\Omega}^{\prime}\right)\left|\overrightarrow{\Omega^{\prime}} \cdot \vec{n}\right| \mathrm{d} \Omega^{\prime}
\end{aligned}
$$

where $\varepsilon_{v}, \rho_{\mathrm{sp}}, \rho_{\mathrm{d}}$ and $\vec{n}$ are the spectral emissivity, specular reflectivity, scattering reflectivity and the outward unit normal vector of the surface, respectively.

This transient problem requires an initial condition. At the beginning $(t=0)$, the sample is at thermal equilibrium at the desired temperature $T_{\infty}$ :

$T(\vec{R}, 0)=T_{\infty} \quad \forall \vec{R}$

Then, the $\mathrm{CO}_{2}$ laser generates a time and space-dependent excitation, entirely absorbed by the first soleplate. All external surfaces exchange with the environment. At high temperature, the major heat loss is due to radiative transfer. In the case of a slight increase in temperature, radiative losses are linearized:

$\varepsilon \sigma\left(T_{\text {ext }}^{4}-T_{\infty}^{4}\right) \simeq 4 \varepsilon \sigma T_{\text {ref }}^{3}\left(T_{\text {ext }}-T_{\infty}\right)=h_{\mathrm{r}}\left(T_{\text {ext }}-T_{\infty}\right)$

Hence, the boundary condition is defined with a total exchange coefficient $h_{\mathrm{t}}=h_{\mathrm{r}}+h_{\text {conv }}$, including both transfers and $T_{\text {ref }}=\left(T_{\text {ext }}+\right.$ $\left.T_{\infty}\right) / 2$ the reference temperature.

The investigated samples were cylindrical (see Fig. 1). Assuming invariance along the angle coordinate, we solved the system

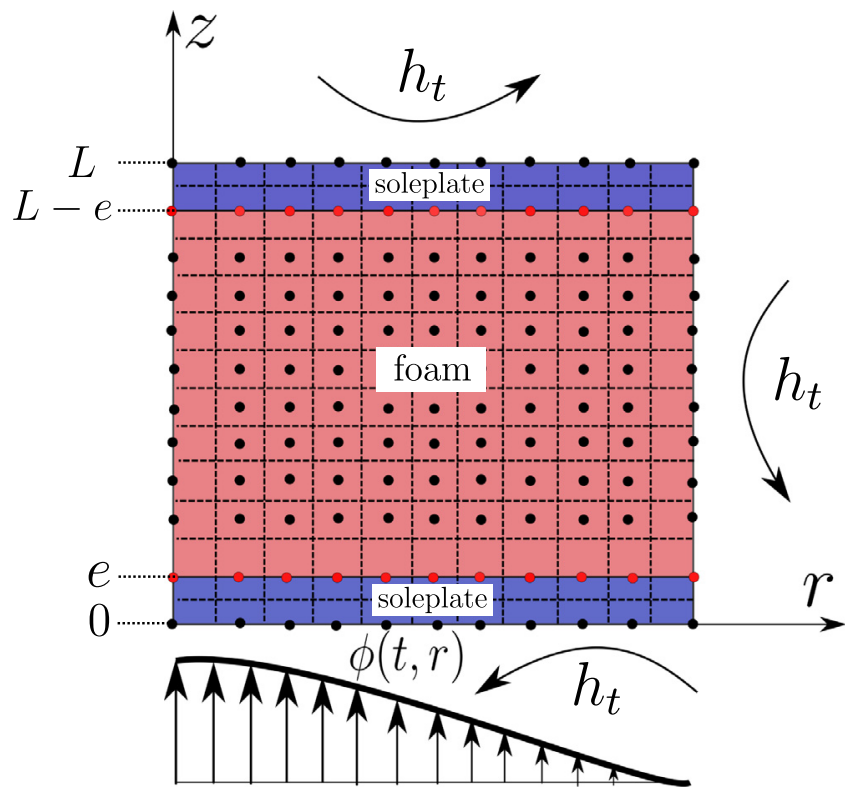

Fig. 1. Flash method multilayer 2-D in cylindrical coordinates system.

of equations in a cylindrical coordinate system with $\vec{R}=r \overrightarrow{e_{r}}+z \overrightarrow{e_{z}}$ where $r$ is the radial coordinate and $z$ the axial coordinate. At both horizontal interfaces between the soleplates and the environment, the heat flux balance leads to

$-\lambda_{\text {sole }}\left(\frac{\partial T}{\partial z}\right)_{z=0}=h_{\mathrm{t}}\left(T(r, 0, t)-T_{\infty}\right)+\phi(r, t) \quad \forall r$

$-\lambda_{\text {sole }}\left(\frac{\partial T}{\partial z}\right)_{z=L}=-h_{\mathrm{t}}\left(T(r, L, t)-T_{\infty}\right) \quad \forall r$

At the interfaces between the soleplates and the foam, we assumed that there was no thermal contact resistance. Heat flux continuity is then written as:

$-\lambda_{\text {sole }}\left(\frac{\partial T}{\partial z}\right)_{\text {sole }, z=e}=-\lambda_{\text {foam }}\left(\frac{\partial T}{\partial z}\right)_{\text {foam }, z=e}+\left(\vec{q}_{\mathrm{r}} \cdot \vec{n}\right)_{z=e} \quad \forall r$

and:

$-\lambda_{\text {sole }}\left(\frac{\partial T}{\partial z}\right)_{\text {sole }, z=L-e}=-\lambda_{\text {foam }}\left(\frac{\partial T}{\partial z}\right)_{\text {foam }, z=L-e}+\left(\vec{q}_{\mathrm{r}} \cdot \vec{n}\right)_{z=L-e} \quad \forall r$

At the lateral side of the cylinder, boundary conditions for the soleplates at the radius $r=R$ are as follows:

$-\lambda_{\text {sole }}\left(\frac{\partial T}{\partial r}\right)_{r=R}=-h_{\mathrm{t}}\left(T(R, z, t)-T_{\infty}\right) \quad \forall z \in[0 ; e] \cap[L-e ; L]$

and for the foam:

$$
\begin{aligned}
& -\lambda_{\text {foam }}\left(\frac{\partial T}{\partial r}\right)_{r=R} \\
& =-h_{\mathrm{t}}\left(T(R, z, t)-T_{\infty}\right)+\left(\vec{q}_{\mathrm{r}} \cdot \vec{n}\right)_{r=R} \quad \forall z \in[e ; L-e]
\end{aligned}
$$

\subsection{Models}

The set of equations described above is solved with two different methods. The first one, applied to the EBE, is deterministic, 


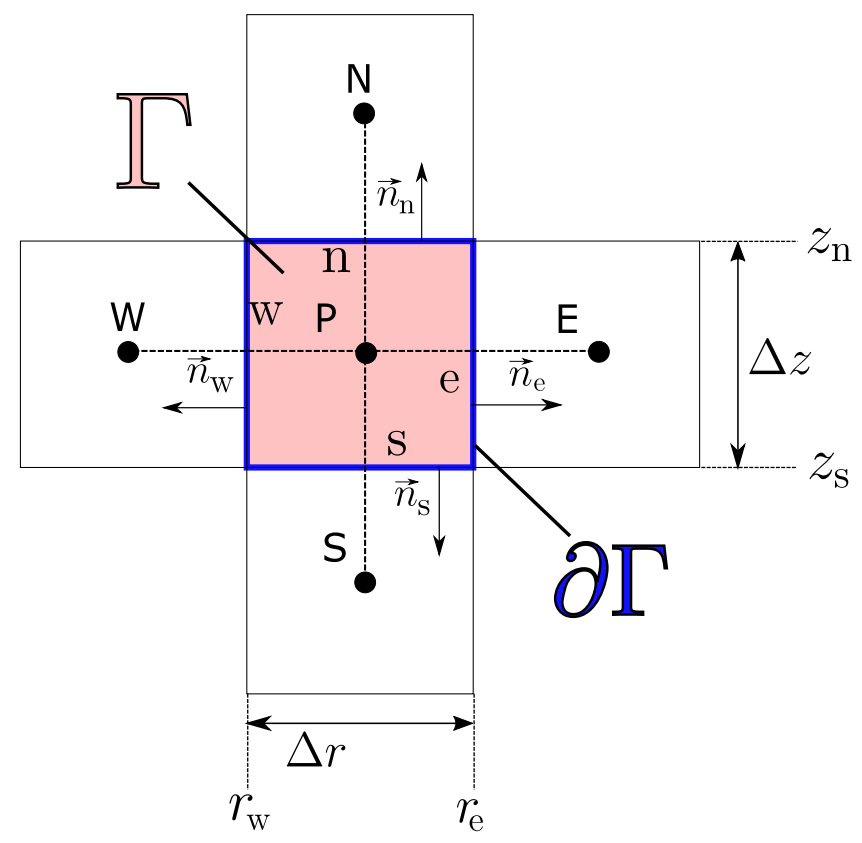

Fig. 2. Control Volume for volumic nodes.

while the second one, applied to the RTE, is stochastic. The principle is to compute the temperature field at a discretized time $t^{k}$ with a finite volume method. Then, the temperature field is transmitted as an input to the RTE solver. A Monte-Carlo method estimates the energy exchanges due to the radiative interaction and returns the radiative source term for all volume and surface elements. Finally, the temperature is computed at a new time step $t^{k+1}$. This procedure, which aims to evaluate a radiation intensity field from the previous time step, corresponds to a weak coupling between EBE and RTE is a commonly made assumption [40].

\subsubsection{Solving the energy balance equation}

In order to solve the EBE, the domain $\Gamma$ is discretized into $N_{r} \times N_{z}$ control volume nodes. As shown in Fig. 2, we developed a cell-centered scheme based on a structured mesh. The temperature is assumed constant in the control volume. The Finite Volume Method is based on the calculation of the EBE at each node. For the nodes included in the semi-transparent medium, Eq. (4) is rewritten as:

$\int_{\Gamma} \rho C_{p} \frac{\partial T}{\partial t} \mathrm{~d} \Gamma=-\left(\int_{\Gamma} \vec{\nabla} \cdot \overrightarrow{q_{\mathrm{c}}} \mathrm{d} \Gamma-\int_{\Gamma} S_{\mathrm{r}} \mathrm{d} \Gamma\right)$

and with the application of Green-Ostrograski theorem:

$\int_{\Gamma} \rho C_{p} \frac{\partial T}{\partial t} \mathrm{~d} \Gamma=-\left(\int_{\partial \Gamma} \overrightarrow{q_{\mathrm{c}}} \cdot \vec{n} \mathrm{~d}(\partial \Gamma)-\int_{\Gamma} S_{\mathrm{r}} \mathrm{d} \Gamma\right)$

where $\partial \Gamma$ is the contour of the elementary domain $\Gamma$. If we integrate over the surface, we obtain:

$$
\begin{gathered}
\pi\left(r_{\mathrm{e}}^{2}-r_{\mathrm{w}}^{2}\right) \Delta z \rho C_{p} \frac{\partial T}{\partial t}=2 \pi\left(r_{\mathrm{w}} q_{\mathrm{w}}^{r}-r_{\mathrm{e}} q_{\mathrm{e}}^{r}\right) \Delta z \\
+\pi\left(r_{\mathrm{e}}^{2}-r_{\mathrm{w}}^{2}\right)\left(q_{\mathrm{s}}^{z}-q_{\mathrm{n}}^{z}\right)+\pi\left(r_{\mathrm{e}}^{2}-r_{\mathrm{w}}^{2}\right) \Delta z S_{\mathrm{r}, \mathrm{P}}
\end{gathered}
$$

All boundary conditions appear in the heat flux term $q_{\mathrm{m}}^{\mathrm{l}}$ with $1=(r, z)$ and $m=(w, e, n, s)$. The detailed set of equations is not given for the sake of readability. Then, a first order backward timestep discretization (Implicit Euler scheme) was chosen. For an in- ternal node with no boundary condition, the following relation can be written:

$$
\begin{aligned}
& \frac{T_{\mathrm{P}}^{k+1}-T_{\mathrm{P}}^{k}}{\Delta t}=\frac{2 a}{\Delta r\left(r_{\mathrm{e}}^{2}-r_{\mathrm{w}}^{2}\right)}\left(r_{\mathrm{e}}\left(T_{\mathrm{E}}^{k+1}-T_{\mathrm{P}}^{k+1}\right)-r_{w}\left(T_{\mathrm{P}}^{k+1}-T_{\mathrm{W}}^{k+1}\right)\right) \\
& \quad+a\left(\frac{T_{\mathrm{N}}^{k+1}-T_{\mathrm{P}}^{k+1}}{\Delta z^{2}}-\frac{T_{\mathrm{P}}^{k+1}-T_{\mathrm{S}}^{k+1}}{\Delta z^{2}}\right)+\frac{1}{\rho C_{p}} S_{\mathrm{r}, \mathrm{P}}^{k}
\end{aligned}
$$

where $a=\lambda / \rho C_{p}$ is the thermal diffusivity. Hence, all nodes are scanned and the associated thermal value (soleplate or foam) is attributed. Finally, the temperature field at the time step $k+1$ is estimated by solving the set of equations such as Eq. (21) at each node.

\subsubsection{Solving the radiative transfer equation}

The term $S_{\mathrm{r}, i}$, quantifying the radiation interaction in the cell $i$, is computed using an improved Monte-Carlo Method (MCM). During the past decade, the MCM appeared to be one of the reference methods for solving the RTE. The main idea of this method is to send a large amount of "Energy carriers", called quanta, to compute the radiative exchange between the cells. In Eq. (7), the first term represents the energy sent from a cell $i$ to the rest of the domain (emission of radiation by the cell) while the second term corresponds to the energy provided to the cell $i$ (absorption of radiation by the cell). For the sake of simplicity, the spatial discretization is the same as that described in the previous section (Section 2.2.1). Moreover, if $i$ is the cell in which the radiative source term is computed, $j$ represents all the other cells of the domain. Hence, the energy balance of a cell $i$ of volume $V_{i}$ is determined as:

$S_{\mathrm{r}, i} V_{i}=\sum_{j=0}^{N} \int_{v_{\min }}^{v_{\max }}\left(P_{i j, v}^{\mathrm{ea}}-P_{j i, v}^{\mathrm{ea}}\right) \mathrm{d} v=P_{i}^{\mathrm{e}}-\sum_{j=0}^{N} \int_{v_{\min }}^{v_{\max }} P_{j i, v}^{\mathrm{ea}} \mathrm{d} v$

where $P_{i j . v}^{\text {ea }}$ is the spectral radiative power emitted by the cell $i$ and absorbed by the cell $j$, and $P_{i}^{\mathrm{e}}$ is the total radiative power, emitted by the cell $i$. The frequencies $v_{\min }$ and $v_{\max }$ were chosen to take into account $99 \%$ of the emission of the blackbody. Eq. (22) simply expresses the balance between the energy emitted and absorbed in one cell. For a boundary element of surface $S_{i}$, the net radiative flux exchanged at the interface is computed as:

$\left(\vec{q}_{\mathrm{r}} \cdot \vec{n}\right)_{i} S_{i}=\sum_{j=0}^{N} \int_{v_{\min }}^{v_{\max }}\left(P_{i j, v}^{\mathrm{ea}}-P_{j i, v}^{\mathrm{ea}}\right) \mathrm{d} \nu=P_{i}^{\mathrm{e}}-\sum_{j=0}^{N} \int_{v_{\min }}^{v_{\max }} P_{j i, v}^{\mathrm{ea}} \mathrm{d} \nu$

The emission power of a volume element $i$ is computed as:

$P_{i V}^{\mathrm{e}}=\int_{0}^{+\infty} \int_{V} 4 \pi \kappa_{\mathrm{a} v} I_{v}^{\mathrm{bb}}\left(T_{i}\right) \mathrm{d} V \mathrm{~d} v$

whereas the power emitted at a boundary element is expressed as:

$P_{i S}^{\mathrm{e}}=\int_{0}^{+\infty} \int_{S} \pi \varepsilon_{v} I_{v}^{\mathrm{bb}}\left(T_{i}\right) \mathrm{d} S \mathrm{~d} v$

Each quantum carries the same fraction of this power, and is attenuated in the medium according to the Beer-Lambert law. Hence, the power exchanged between the $n q^{\text {th }}$ quantum emitted by $i$ and the crossed cell $j$ is:

$P_{i j, v, n q}^{\text {exch }}=P_{\mathrm{q}}\left(\vec{R}_{1}\right)-P_{\mathrm{q}}\left(\vec{R}_{2}\right)=P_{\mathrm{q}}\left(\vec{R}_{1}\right)\left(1-\exp \left(-\kappa_{\mathrm{a} v} \Delta R\right)\right)$

where $\vec{R}_{1}$ and $\vec{R}_{2}$ are the entry and exit position of the crossed cell, respectively, $P_{\mathrm{q}}$ is the carried power, and $\Delta R$ is the distance travelled through the cell $j$. The power lost by the $n q^{\text {th }}$ quantum corresponds to a power gain for the cell $j$. Let us note that in such a cylindrical coordinates system, a cell takes the form of a ring with a square section. 
This approach is called Forward Method (FM). Improved MonteCarlo Methods make it possible to greatly reduce the number of quanta needed to achieve a given precision. Hence, based on the radiation reciprocal principle, which includes aspect ratio and radiative energetic exchange, Absorption Reciprocity Method (ARM) and Emission Reciprocity Method (ERM) were proposed [41]. However, it was demonstrated that some discrepancies are possible while computing the divergence term in cold regions of the domain. Hence, Zhang et al. [42] proposed an extended method, named Optimized Emission Reciprocity Method (OERM), based on a preferential random sampling of the spectral distribution of the hottest cell of the domain. We chose this formulation for our study. Then, the divergence of the radiative flux can be computed according to:

$S_{\mathrm{r}, i}^{\mathrm{OERM}} V_{i}=P_{i}^{\mathrm{e}}\left(T_{\max }\right) \sum_{j=0}^{N} \int_{v_{\min }}^{v_{\max }}\left(1-\frac{I_{v}^{\mathrm{bb}}\left(T_{j}\right)}{I_{v}^{\mathrm{bb}}\left(T_{i}\right)}\right)\left(\frac{I_{\nu}^{\mathrm{bb}}\left(T_{i}\right)}{I_{\nu}^{\mathrm{bb}}\left(T_{\max }\right)}\right) \mathrm{d} \nu$

where $P_{i}^{\mathrm{e}}\left(T_{\max }\right)$ is the total power, emitted by the highest temperature of the domain. The methods based on the emission are very convenient. Indeed the term $S_{\mathrm{r}, i}$ can be computed with quanta sent from $i$ alone. Unlike methods based on absorption, it is no longer necessary to scan all the cells, to compute all the radiative source, each can be calculated one after the other. Moreover, we can impose a convergence criterion for each cell, in order to control the accuracy of the estimated value. Hence, the number of realizations is not fixed. When this criterion is reached, the algorithm evaluates the radiative source term in the next cell. The procedure ends when the radiative source term is computed in all cells.

\subsubsection{Inversion method: Levenberg-Marcquardt algorithm}

The inversion method principle is based on the fitting of the temporal evolution of the temperature $T_{\text {mod }}(t)$ on the rear face $(z=L)$, given by the model, and the corresponding data $T_{\exp }(t)$ measured experimentally. The main idea is to minimize the cost function $J$, which quantifies the sum of the quadratic differences between the two curves:

$J=\sum_{k=1}^{N_{t}}\left(T_{\exp }\left(t^{k}\right)-T_{\bmod }\left(t^{k}\right)\right)^{2}$

The temporal evolution given by the model depends on a set of parameters $\alpha=\left[\alpha_{1}, \ldots, \alpha_{n}, \ldots, \alpha_{N}\right]$. Hence, any of the quantities defining the geometry, properties of the media and all predisposed assumptions have an impact on the final result. The principle of the inversion is to determine the optimal set of parameters, which provides the best match:

$J_{\min }=J\left(\alpha_{\text {opt }}\right)=\sum_{k=1}^{N_{t}}\left(T_{\text {exp }}\left(t^{k}\right)-T_{\text {mod }}\left(\alpha_{\text {opt }}, t^{k}\right)\right)^{2}$

The system to solve in order to optimize the set of parameters is:

$\frac{\partial J_{\min }}{\partial \alpha_{n}}\left(\alpha_{\text {opt }}\right)=\sum_{k=1}^{N_{t}}\left[\left(T_{\exp }\left(t^{k}\right)-T_{\bmod }\left(\alpha_{\text {opt }}, t^{k}\right)\right) \frac{\partial T_{\bmod }}{\partial \alpha_{n}}\right]=0$

which can also be written as:

$\alpha_{\mathrm{opt}}=\left(\underline{\underline{S}}^{T} \underline{\underline{S}}\right)^{-1} \underline{\underline{S}}^{T}\left(T_{\exp }-T_{\bmod }\right)$

where $\underline{\underline{S}}$ is the sensitivity matrix. The sensitivity of a parameter $\alpha_{n}$ is computed with a centered scheme:

$$
\begin{aligned}
& S\left(\alpha_{n}, t\right)=\frac{\partial T_{\bmod }\left(\alpha_{1}, ., \alpha_{n}, ., \alpha_{N}, t\right)}{\partial \alpha_{n}} \simeq \\
& \frac{T_{\bmod }\left(\alpha_{1}, ., \alpha_{n}+\mathrm{d} \alpha_{n}, ., \alpha_{N}, t\right)-T_{\bmod }\left(\alpha_{1}, ., \alpha_{n}-\mathrm{d} \alpha_{n}, ., \alpha_{N}, t\right)}{2 \mathrm{~d} \alpha_{n}}
\end{aligned}
$$

However, due to the non-linearity of the studied problem, the computation of the optimum set of parameters is obtained itera- tively. Eq. (31) then becomes:

$\alpha^{p+1}=\alpha^{p}+\left(\underline{\underline{S}}^{T}\left(\alpha^{p}\right) \underline{\underline{S}}\left(\alpha^{p}\right)+\mu^{p} \underline{\underline{D}}^{p}\right)^{-1} \underline{\underline{S}}^{T}\left(\alpha^{p}\right)\left(T_{\exp }-T_{\bmod }\left(\alpha^{p}\right)\right)$

where $\mu^{p}$ is a relaxation coefficient, $\underline{D}^{p}$ is a diagonal matrix and $p$ the interation index of the inversion procedure. Eq. (33) is known as the Levenberg-Marcquard method [43].

The inversion algorithm is stopped when the cost function between two iterations $\left|J^{p+1}-J^{p}\right|$ is lower than the convergence criterion.

\subsubsection{Parameters selection}

At each iteration, the so-called information matrix $\left(\underline{\underline{S}}^{T}\left(\alpha^{p}\right) \underline{\underline{S}}\left(\alpha^{p}\right)\right)$ is inversed. Moreover, when the algorithm has converged, the uncertainty on the estimated red value is given by:

$\sigma_{\mathrm{b}}^{2}\left(\underline{\underline{S}}^{T} \underline{\underline{S}}\right)^{-1}=\left(\begin{array}{cccc}\operatorname{var}\left(\alpha_{1}\right) & \operatorname{cov}\left(\alpha_{1}, \alpha_{2}\right) & \ldots & \operatorname{cov}\left(\alpha_{1}, \alpha_{N}\right) \\ \operatorname{cov}\left(\alpha_{1}, \alpha_{2}\right) & \operatorname{var}\left(\alpha_{2}\right) & \ldots & \operatorname{cov}\left(\alpha_{2}, \alpha_{N}\right) \\ \vdots & \vdots & \ddots & \vdots \\ \operatorname{cov}\left(\alpha_{1}, \alpha_{N}\right) & \operatorname{cov}\left(\alpha_{2}, \alpha_{N}\right) & \ldots & \operatorname{var}\left(\alpha_{N}\right)\end{array}\right)$

where $\sigma_{\mathrm{b}}$ is the standard deviation of the noise, $\operatorname{var}\left(\alpha_{n}\right)$ is the variance of the $n^{\text {nt }}$ structural parameter of the model and cov is the covariance between two parameters. Hence, a low sensitivity or correlation between two parameters could lead to a bad conditioning of the matrix. This phenomenon causes high uncertainties about results. The strategy in the choice of the set of parameters is thus very important.

In order to avoid multiple correlations, the number of parameters has to be as low as possible. Geometric properties, such as the radius and the thickness of the sample and of the soleplates were precisely measured. Moreover, thermal properties of the soleplates were also characterized with complementary methods. Thermal diffusivity $a_{\text {sole }}$ was measured with an adapted flash method, and $\left(C_{p}\right)_{\text {sole }}$ with a Differential Scanning Calorimetry (DSC) apparatus (multi-HTC 96 from Setaram ${ }^{\circledR}$ ). Moreover, a sensitivity analysis showed that the heat capacity of the foam is strongly correlated with its equivalent diffusivity. $\left(C_{p}\right)_{\text {bulk }}$ was also measured with the DSC method. All of these properties were estimated for a wide range of temperature ( $293 \mathrm{~K}$ to $1373 \mathrm{~K}$ ). The equivalent heat capacity of the foam is related to the heat capacity of the ceramic $\left(C_{p}\right)_{\text {bulk, according to: }}$

$\left(\rho C_{p}\right)_{\text {foam }}=(1-\epsilon)\left(\rho C_{p}\right)_{\text {bulk }}$

where $\epsilon$ is the foam porosity. Thus, this porosity must also be known as well. In order to measure it precisely, we used a pycnometer [44], which allows measurement of open porosity.

Concerning the equivalent radiative properties of the foam, is should be noted that the proposed procedure does not investigate the validity of the Beerian behavior of the equivalent porous medium. Indeed, it should be remembered that the idea of this study is to experimentally estimate Beerian radiative properties that are apparent or effective. Moreover, the RDFI method [29] was applied to a few samples and the results (not shown) indicated that the assumption of the Beerian behavior seems satisfactory as long as the heterogenous medium is composed of an opaque phase and a transparent phase. This part of our experimentation is not presented here for the sake of brevity and will be the subject of future studies.

Estimation of equivalent wavelength-dependent radiative properties seems very complicated to perform because the number of parameters would become too large, even using of a physical law such as Drude-Lorentz. Therefore, we considered one global radiative property. This does not mean that the radiative property is in- 
dependent on the wavelength, but that the estimated value represents an equivalent grey medium and its radiative behavior. This assumption was widely investigated by Coquard et al. [38], who showed that, using a simulated process, a non-grey medium can be considered as a grey medium.

Several studies $[37,45]$ have previously demonstrated that scattering effects could have the same influence on the final result as absorption/emission effects. It thus becomes difficult to separate the influence of absorption phenomena from that of scattering phenomena (correlation of the two parameters), so we instead identified only the equivalent optical thickness related to the extinction coefficient and to the thickness of the medium such as $\tau=\beta e_{\text {foam }}$. Furthermore, considering an equivalent homogeneous medium, scattering events are only due to the reflectivity of the bulk such as $\rho_{\text {bulk }}=\kappa_{\mathrm{d}} / \beta$ [33]. An analysis of the optical surface properties of the $\mathrm{SiC}$ revealed that this material is highly absorbing. An estimation of the scattering coefficient and the phase function parameter $\Phi_{\nu}\left(\overrightarrow{\Omega^{\prime}} \rightarrow \vec{\Omega}\right)$ is impossible due to the low influence of scattering phenomena. Hence, we set the value of the scattering coefficient to zero.

The spatial and time dependence of the excitation could introduce large uncertainties about the result. Hence, the duration of the excitation was measured. Simulations, taking into account several widths of collimated beams, showed that the excitation can be assumed to be spatially homogeneous due to the high diffusivity of the soleplates. We also precisely identified the maximum of the experimental curve $T_{\max }$ by fitting it with a polynomial. The maximum temperature therefore corresponds to the maximum of the fitted curve. Moreover, a normalized temperature, that avoids knowing what energy is brought to the system (sample + soleplates) by the laser, is defined according to:

$\theta_{\text {mod }}(t)=K\left(\frac{\bar{T}(t)-\bar{T}_{\min }}{\bar{T}_{\max }-\bar{T}_{\min }}\right)$

where $\bar{T}$ is the spatial average of the rear surface temperature and $K$ is an adjustment parameter. Very close to unity, the latter makes it possible to correct the maximum of the curve obtained by the model and thus decreases the discrepancies due to experimental noise. Moreover, for the same reasons, working from an average temperature in space logically allows better performance than from a single spatial value.

In summary, we kept a set of four parameters $\left[a_{\text {foam }}, h_{\mathrm{t}}, K, \tau\right]$, which correspond to the equivalent diffusivity of the foam due only to conduction transfers, the total convection coefficient, the adjustment parameter and the optical thickness, respectively. Thus just one thermal $a_{\text {foam }}$ and one radiative parameter $\tau$ are sufficient to reproduce the thermogram obtained with the foams. The two other parameters are simply extrinsic parameters.

From the identified equivalent diffusivity we extract the equivalent conductivity:

$\lambda_{\text {foam }}=a_{\text {foam }}(1-\epsilon)\left(\rho C_{p}\right)_{\text {bulk }}$

Additionaly we made some adjustments using a purely conductive model with only one thermal parameter, which thus gives an equivalent total conductivity including an apparent radiative conductivity: $\lambda_{\text {tot }}=\lambda_{\text {foam }}+\lambda_{\text {rad }}$. When the optical thickness of the sample is not too large, the inversion with the two-parameter model $\left(a_{\text {foam }}, \tau\right)$ is better than the one-parameter model (only $a_{\mathrm{tot}}$ ). In this case the sensitivity to the optical thickness is sufficient to identify it. On the contrary, for high optical thicknesses, the radiation transfer can be modelled by a simple diffusion like the thermal transfer (Rosseland model). Due to correlation effect, it becomes difficult to separate the conduction from the radiation.

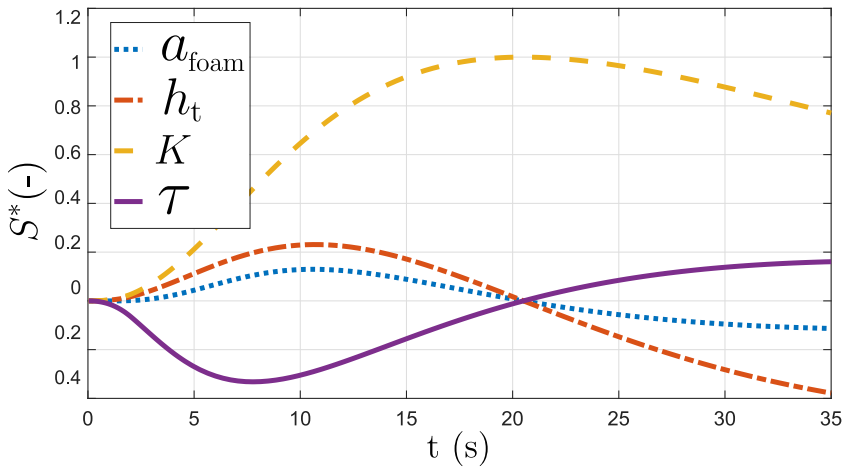

Fig. 3. Sensitivity analysis for $\tau=2.5$.

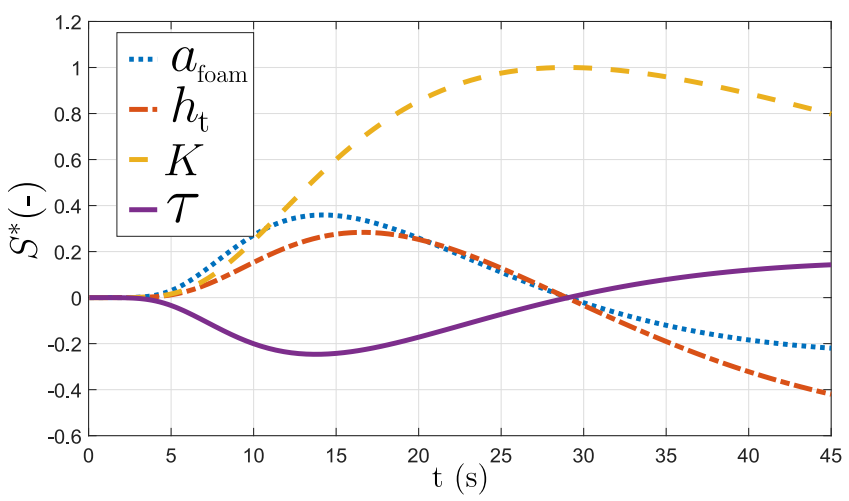

Fig. 4. Sensitivity analysis for $\tau=10$.

\subsubsection{Parameter identifiability}

In order to verify the impact of the different parameters and to quantify the error of the estimation due to experimental noise, it is common to simulate fictive experiments. We investigated cases of optically thin (Case 1: $\tau=2.5$ ) and thick (Case 2: $\tau=10$ ) media. The size of the sample and the temperature were set at $R=$ $25 \mathrm{~mm}, e=20 \mathrm{~mm}$ and $T_{\infty}=1073 \mathrm{~K}$. The diffusivity of the foam, total convection coefficient and normalization parameter were set at: $a_{\mathrm{foam}}=3 \mathrm{~mm}^{2} \mathrm{~s}^{-1}, h_{\mathrm{t}}=150 \mathrm{~W} \mathrm{~m}^{-2} \mathrm{~K}^{-1}, K=1$. The corresponding thermal conductivity was that of an insulating foam with $\lambda_{\text {foam }}=1 \mathrm{~W} \mathrm{~m}^{-1} \mathrm{~K}^{-1}$. A white noise with a standard deviation of $\sigma_{\mathrm{b}}=0.5 \%$ was added. Results of the inversion are given in the Table 1.

In both thin and thick examples, the inversion algorithm retrieves the chosen parameters well. In Figs. 3 and 4 the reduced sensitivities $S_{n}^{*}=\alpha_{n}\left(\partial \theta / \partial \alpha_{n}\right)$ are represented according to time. These make it possible to compare the influence of the different parameters. In case 1, one can see (see Fig. 3) that the sensitivity associated with optical thickness reaches its maximum before the sensitivity of diffusivity. This difference is explained by a higher speed of propagation of radiation through the sample compared with conduction. Both conductive and radiative sensitivities are non-zero and each of them acts at a different time. However, this does not guarantee the accurate estimation of all parameters in the case of the optically thick foam (see Fig. 4). The radiative transfer plays the same role as the conductive transfer. Therefore, both transfers have the same impact on the final curve and it becomes difficult to separate one from the other during the inversion process. This introduces uncertainties about the correlated parameters. Thus, using Eq. (34) we find uncertainties of $3.3 \%$ for the diffusivity and $5.8 \%$ for the optical thickness. For case 1 , there is no correlation, however, since the radiative transfer is dominant, the accuracy obtained for the conductive parameter is lower than that of the optical thickness. We note an uncertainty of $1.34 \%$ for 
Table 1

Cases investigated.

\begin{tabular}{lllll}
\hline Case & $a_{\text {foam }}\left(\mathrm{mm}^{2}\right)$ & $h_{\mathrm{t}}\left(\mathrm{W} \mathrm{m}^{-2} \mathrm{~K}^{-1}\right)$ & $K(-)$ & $\tau(-)$ \\
\hline 1 & $2.996( \pm 1.34 \%)$ & $149.99( \pm 1.4 \%)$ & $2.497( \pm 0.23 \%)$ & $1.000( \pm 0.03 \%)$ \\
2 & $2.943( \pm 3.3 \%)$ & $150.56( \pm 0.63 \%)$ & $9.771( \pm 5.8 \%)$ & $1.000( \pm 0.03 \%)$ \\
\hline
\end{tabular}

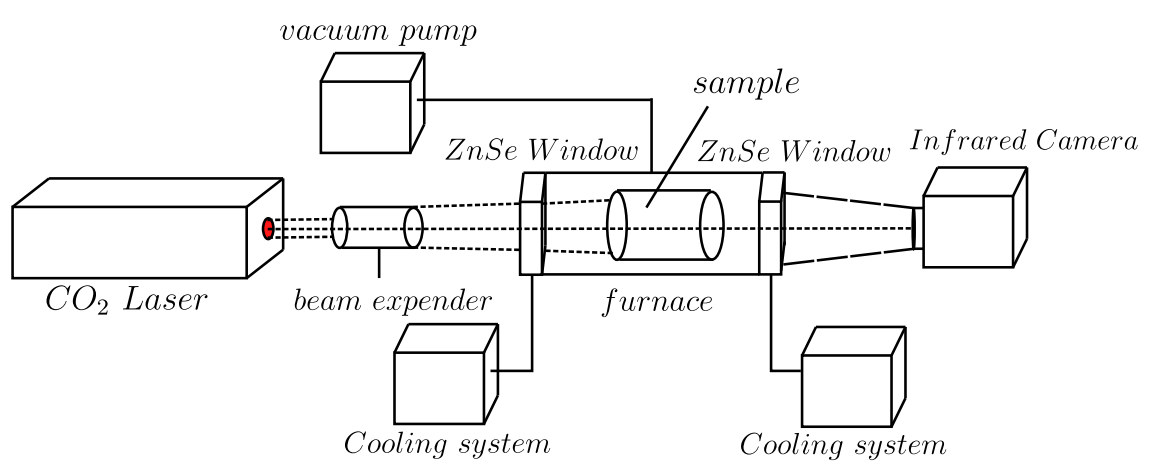

Fig. 5. Experimental apparatus.

the first parameter and $0.23 \%$ for the optical thickness. We should draw attention to the fact that this uncertainty represents only the part coming from the inversion process itself and is therefore not a total uncertainty. There are other sources of uncertainty such as the accuracy associated with the model, the assumptions made, the experimental implementation or the parameters assumed to be known and fixed.

\section{Experimental investigations}

\subsection{Experimental set-up}

The experimental set-up is close to the flash method experiment, explained by Shick et al. [46]. Some modifications were made in order to enable the study of foams. A diagram showing the different parts of the experimental apparatus is shown in Fig. 5. The source is a $\mathrm{CO}_{2}$ laser ( $130 \mathrm{~W}$ Synrad) with a wavelength of $10.6 \mathrm{~mm}$. This emits a beam of $2.1 \mathrm{~mm}$ diameter which is expanded to $8.4 \mathrm{~mm}$ by using a two-lens afocal optical system. The laser emits at a constant power over a fixed time duration. Two $\mathrm{ZnSe}$ windows are positioned at the entrance and exit of the furnace. The first of these windows has an anti-reflective coating, which improves the heat flux transmitted to the sample. Both windows are cooled with a water system at ambient temperature. A vacuum pump maintains a pressure of $9.10^{-3}$ mbar, measured at the pump inlet. An infrared InSb camera (Flir SC7000 Titanium) with a broadband sensor $(1.5 \mu \mathrm{m}$ to $5.1 \mu \mathrm{m})$ records the temperature field of the rear face of the sample. More precisely, the camera records the intensity emitted here. The so-called "Digital Level (DL)" given by the camera is linear with the radiant intensity. Because the temperature variation of the sample is very small in a flash experiment (only a few $\mathrm{K}$ ), it can be also assumed that the Digital Level given by the camera is also linear with the sample temperature. Because only the temporal shape of temperature variation of the sample is necessary in the flash method, the normalized temperature $\theta_{\exp }$ is used:

$\theta_{\exp }(t)=\frac{\bar{T}(t)-\bar{T}_{\min }}{\bar{T}_{\max }-\bar{T}_{\min }}=\frac{\overline{D L}(t)-\overline{D L}_{\min }}{\overline{D L}_{\max }-\overline{D L}_{\min }}$

In this way no temperature calibration of the camera is necessary. At each time step, we computed the space averaged temperature. Then, we obtained the normalized temperature used for the inversion procedure. A tubular Carbolite TZF600 furnace provides a homogeneous temperature in the enclosure. The sample holder is

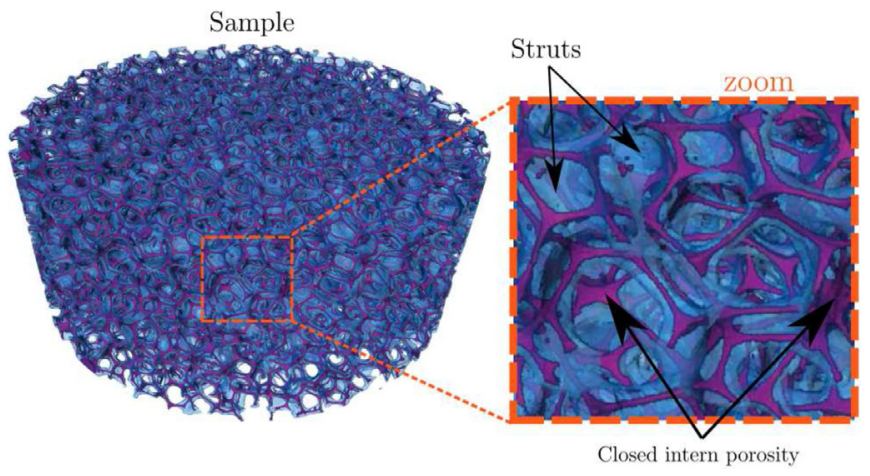

Fig. 6. Tomography-X analysis applied to a random foam, solid is slightly transparent and internal void is opaque.

made of a piece of stumatite. It is composed of two stackable parts and allows a good thermal contact between the two soleplates and the foam. Two different types of soleplates were used: Titanium $\mathrm{Ta} 6 \mathrm{~V}$ and SiC. Samples were chosen in order to investigate three different aspects:

- Influence of the pore diameter

- Difference between structured and random foams

- Influence of the strut material

All the foams studied and their corresponding properties are listed in Table 2. Silicon carbide ( $\mathrm{SiC}$ ) and infiltrated silicon carbide ( $\mathrm{SiSiC}$ ) were considered. Moreover, we studied three different geometries. The fabrication process consists of impregnating a polyurethane foam (PU) with a polysilane slip (ceramic slurry). Then, during sintering, the polymer foam is destroyed and a ceramic skeleton is obtained. It is well-known that the removal of the PU matrix by heat treatment causes a closed porosity to form in the struts. Moreover, this was clearly highlighted with an X-ray tomography analysis. By making the solid slightly transparent, Fig. 6 shows this internal network. For a random foam, $4 \%$ of the measured porosity arises from the internal porosity, which is significant.

Random foams, are represented in Fig. 7 a). Structured (tetra) foams are based on a repetition of the well-known Kelvin-cell (Tetrakaidecahedron), shown in Fig. 7b). Strut length of the structured foams (cubic) is $7 \mathrm{~mm}$ with a pore diameter $10 \mathrm{~mm}$ (Fig. 7c)). In this latter foam, the small diameter "canal" due to 
Table 2

Investigated foams

\begin{tabular}{lllllll}
\hline Foam number & Solid phase & Geometry & Porosity & $D_{\text {cell }}(\mathrm{mm})$ & PPI & $e_{\text {foam }} \mathrm{mm}$ \\
\hline 1 & $\mathrm{SiSiC}$ & Structured (tetra) & 0.84 & 10 & $/$ & 20 \\
2 & $\mathrm{SiSiC}$ & Structured (tetra) & 0.83 & 7.5 & $/$ & 20 \\
3 & $\mathrm{SiSiC}$ & Structured (tetra) & 0.89 & 5 & $/$ & 20 \\
4 & $\mathrm{SiSiC}$ & Structured (cubic) & 0.8 & 10 & $/$ & 20 \\
5 & $\mathrm{SiSiC}$ & Structured (cubic) & 0.8 & 10 & $/$ & 15 \\
6 & $\mathrm{SiC}$ & Unstructured (random) & 0.83 & 10 & 7 & 20 \\
7 & $\mathrm{SiC}$ & Unstructured (random) & 0.82 & 5 & 15 & 20 \\
8 & $\mathrm{SiC}$ & Unstructured (random) & 0.84 & 2.5 & 30 & 20 \\
9 & $\mathrm{SiC}$ & Unstructured (random) & 0.89 & 1.8 & 40 & 20 \\
10 & $\mathrm{SiC}$ & Unstructured (random) & 0.9 & 1.4 & 50 & 20 \\
\hline
\end{tabular}

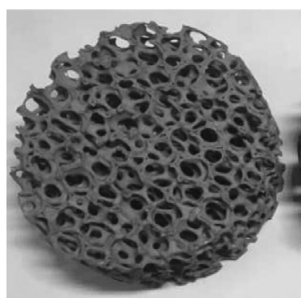

a)

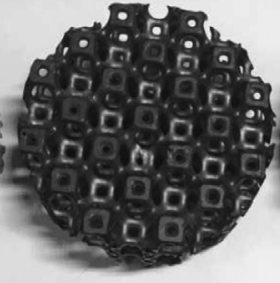

b)

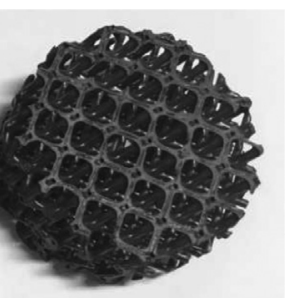

c)
Fig. 7. (a) Random, (b) Structured (tetra) and (c) Structured (cubic) foams.

the manufacturing process is visible in the center of the struts. The structural property of the random foam is characterized by the number of Pores Per Inch (PPI), given by the manufacturer. Although the manufacturer also provided an average cell size of the foam cells associated with the number of PPI, this is not precisely measured. Structured foams, on the other hand, are simply defined by the size of the pore.

\subsection{Experimental results}

Knowing that ceramic foams are commonly used for high temperature applications, we chosed to study their thermal behavior up to $1073 \mathrm{~K}$. For each thermogram obtained, we applied the inversion procedure and estimated the set of parameters, as presented in Section 2.2.4. Figs. 8-10 show, for structured and random foams, the experimental thermograms along with results from the model. All the estimated values are presented in Table 3 and represented in Figs. 11 and 12. Foam number 5 was studied in a temperature range between $[673 \mathrm{~K} ; 1073 \mathrm{~K}]$ and the associated results are represented in Fig. 13.

As explained above, the equivalent total conductivity $\lambda_{\text {tot }}$ was obtained with a purely conductive model, and represents the to-

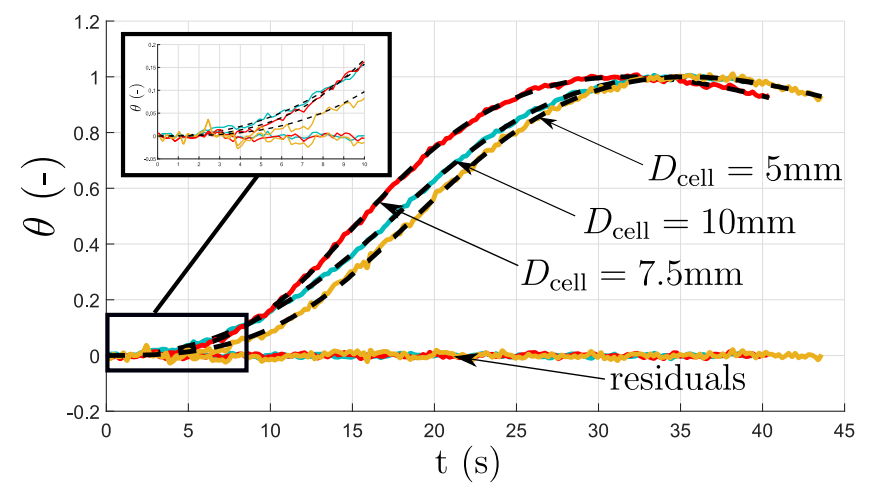

Fig. 8. Structured (tetra) foams at $T_{\infty}=1073 \mathrm{~K}$; experimental results are shwon with continuous lines and the model with dotted lines.

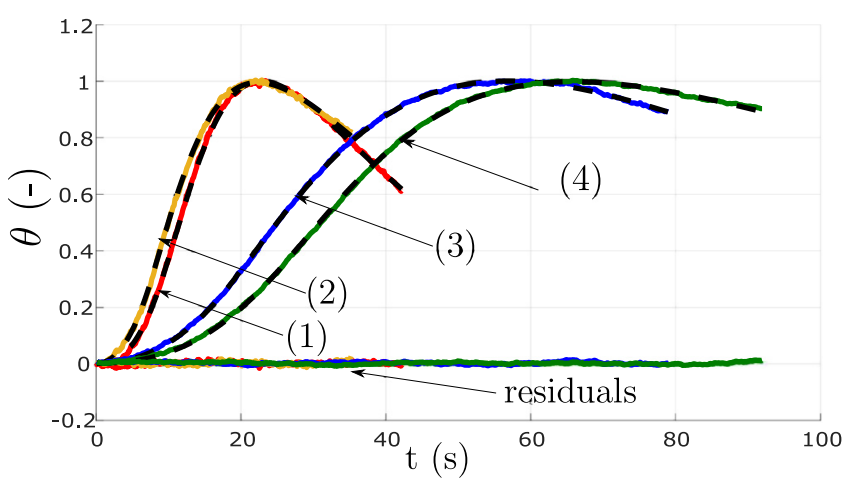

Fig. 9. Structured (cubic) foams: Impact of thickness and temperature, (1) $e=$ $20 \mathrm{~mm} T_{\infty}=1073 \mathrm{~K},(2) e=15 \mathrm{~mm} T_{\infty}=1073 \mathrm{~K}$, (3) $e=15 \mathrm{~mm} T_{\infty}=873 \mathrm{~K}$ and (4) $e=15 \mathrm{~mm} T_{\infty}=773 \mathrm{~K}$; experimental results are shwon with continuous lines and the model with dotted lines.

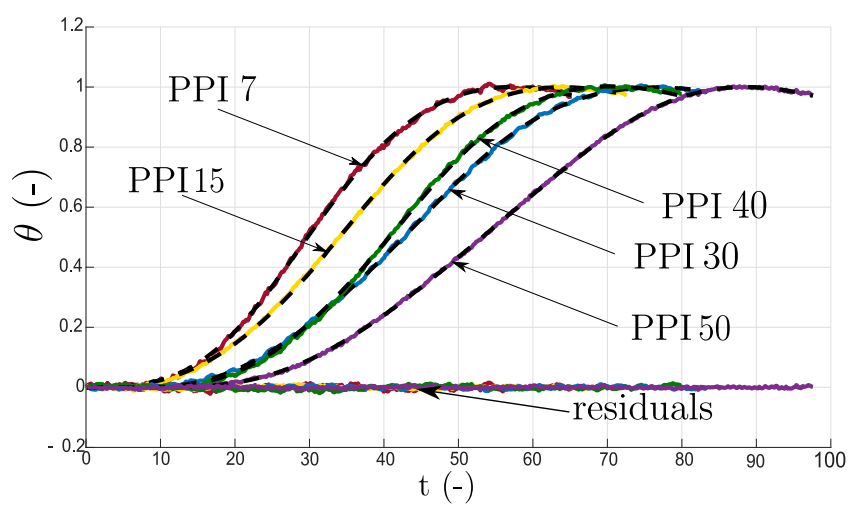

Fig. 10. Random foams at $T_{\infty}=1073 \mathrm{~K}$; experiment in continuous lines and model in dotted lines.

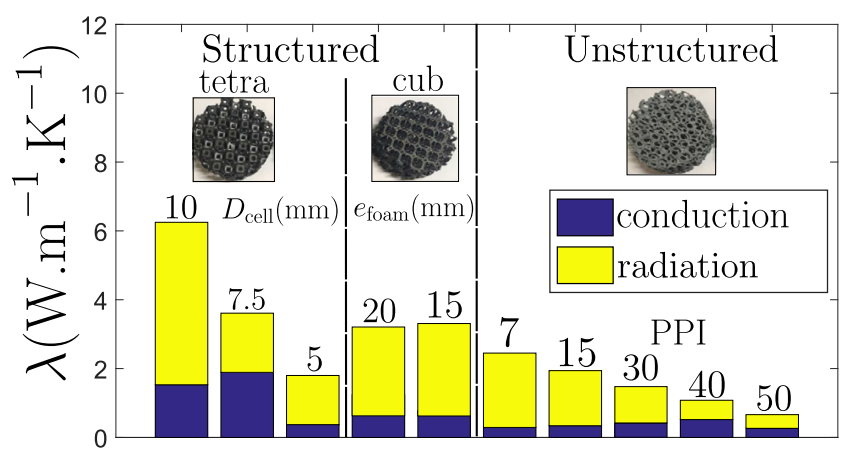

Fig. 11. Conductive and radiative heat transfer of all samples at $T_{\infty}=1073 \mathrm{~K}$. 
Table 3

Identified thermal properties.

\begin{tabular}{|c|c|c|c|c|c|c|c|}
\hline Foam number & $T_{\infty}(\mathrm{K})$ & $\lambda_{\text {tot }}\left(\mathrm{W} \mathrm{m}^{-1} \mathrm{~K}^{-1}\right)$ & $\lambda_{\text {foam }}\left(\mathrm{W} \mathrm{m}^{-1} \mathrm{~K}^{-1}\right)$ & $\lambda_{\text {rad }}\left(\mathrm{W} \mathrm{m}^{-1} \mathrm{~K}^{-1}\right)$ & $\beta\left(\mathrm{mm}^{-1}\right)$ & $\tau(-)$ & $\lambda_{\text {foam }} / \lambda_{\text {tot }}(\%)$ \\
\hline 1 & 1073 & 6.25 & 1.53 & 4.72 & 0.089 & 1.78 & 24.6 \\
\hline 2 & 1073 & 3.61 & 1.89 & 1.72 & 0.212 & 4.24 & 52.4 \\
\hline 3 & 1073 & 1.80 & 0.37 & 1.43 & 0.276 & 5.51 & 20.7 \\
\hline 4 & 1073 & 3.21 & 0.51 & 2.69 & 0.160 & 3.20 & 15.9 \\
\hline 5 & 1073 & 3.50 & 0.54 & 2.96 & 0.142 & 2.13 & 15.7 \\
\hline 5 & 973 & 2.92 & 0.51 & 2.41 & 0.135 & 2.0 & 17.4 \\
\hline 5 & 873 & 2.05 & 0.52 & 1.52 & 0.135 & 2.0 & 25.3 \\
\hline 5 & 773 & 1.68 & 0.54 & 1.15 & 0.135 & 2.0 & 31.9 \\
\hline 5 & 673 & 1.21 & 0.56 & 0.65 & 0.138 & 2.1 & 46.2 \\
\hline 6 & 1073 & 2.45 & 0.29 & 2.16 & 0.199 & 3.98 & 12.1 \\
\hline 7 & 1073 & 1.94 & 0.34 & 1.60 & 0.260 & 5.20 & 17.7 \\
\hline 8 & 1073 & 1.58 & 0.41 & 1.17 & 0.386 & 7.71 & 17.6 \\
\hline 9 & 1073 & 1.08 & 0.52 & 0.56 & 0.613 & 12.26 & 48.2 \\
\hline 10 & 1073 & 0.66 & 0.26 & 0.40 & 0.852 & 17.04 & 39.4 \\
\hline
\end{tabular}

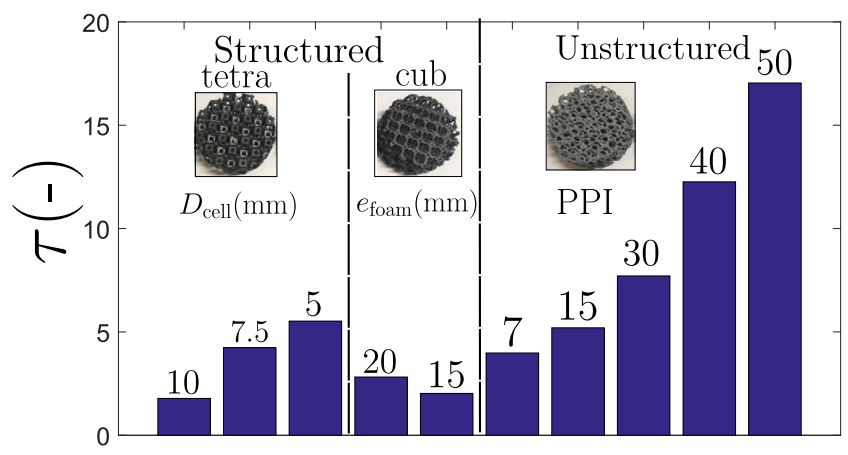

Fig. 12. Optical thickness of all samples at $T_{\infty}=1073 \mathrm{~K}$.

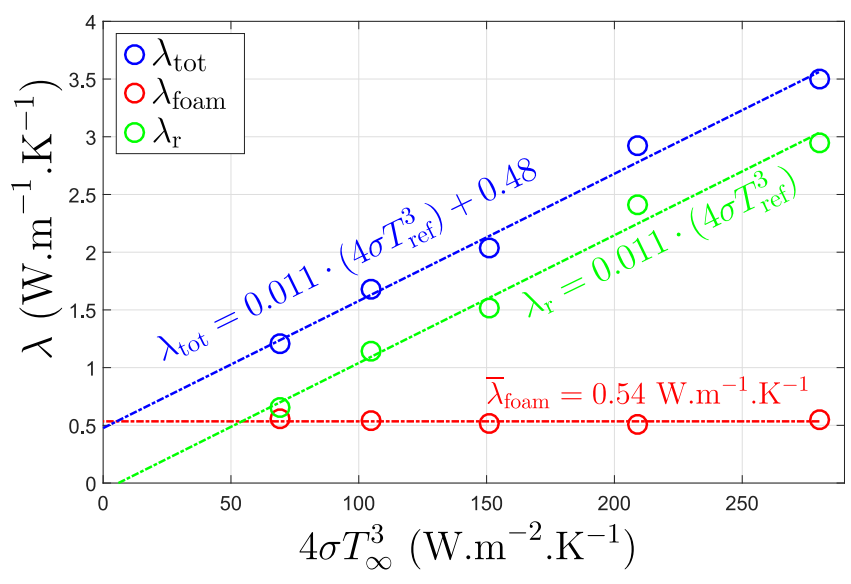

Fig. 13. Structured (cubic) foam $e=15 \mathrm{~mm}$ : equivalent total conductivity, equivalent radiative conductivity and equivalent thermal conductivity.

tal heat flux ( $\lambda_{\text {foam }}+\lambda_{\text {rad }}$ the equivalent conductivity by conduction alone and the equivalent radiative conductivity respectively) through the media. Hence, the difference between the total conductivity and the equivalent conductivity $\lambda_{\text {foam }}$ corresponds to the "radiative" conductivity $\lambda_{\text {rad }}$. The values, which quantify the conductive and radiative heat transfers for all samples at $1073 \mathrm{~K}$ are also represented in Fig. 11 while the estimated optical thickness is indicated in Fig. 12.

The comparisons between experimental and numerical results in Figs. 8-10 show how well the presented direct model agrees with the measurements. This is true when investigating the impact of pore diameter (Figs. 8 and 10), but also the effects of sample thickness and reference temperature (Fig. 9). As demonstrated by Coquard et al. [38], a model based on an effective optical thick- ness describes the measured thermograms very well. The residuals $\theta_{\exp }\left(t^{k}\right)-\theta_{\text {mod }}\left(t^{k}\right)$ are very small and random in all cases.

It should be noted that, as shown in the sensitivity analysis in Section 2.2.5, the smaller the optical thickness, the sooner the temperature rises. However, for the structured foam shown in Fig. 8, the thermogram corresponding to the foam with $D_{\text {cell }}=7.5 \mathrm{~mm}$ reaches its maximum before the thermogram associated with $D_{\text {cell }}=10 \mathrm{~mm}$ or $D_{\text {cell }}=5 \mathrm{~mm}$. This phenomenon is easily explained by the difference of the excitation time. The longer the excitation time, the later the maximum temperature is reached. This is why the thermal excitation time was taken into account in the model. For a structured foam with $D_{\text {cell }}=(5,7.5,10) \mathrm{mm}$, we identified an optical thickness of $\tau=(1.78,4.24,5.51)$ (Fig. 12), respectively. It should be notable that the optical thickness increases threefold when the pore diameter decreases twofold.

More generally, regarding the radiative properties of all foams, the values obtained show that the radiative parameter is very strongly correlated with the pore diameter (see Fig. 12). These results are in good agreement with Coquard et al. [38]. As shown in Fig. 12, optical thickness increases with the number of pores per inch. In other words, optical thickness appears to be proportional to the inverse of the cell diameter. This result can be explained by the fact that the extinction coefficient represents the inverse of the mean free-path. Hence, it corresponds to the inverse of the distance travelled by the radiation before being absorbed. If the pore diameter decreases (increasing PPI), the corresponding optical thickness increases strongly. Hence, at high temperatures, the radiative transfer is so dominating that the increase of the optical thickness has a direct impact on the total equivalent thermal conductivity (see Fig. 11).

Concerning the estimated equivalent conductivity $\lambda_{\text {foam }}$ shown in Fig. 11 and in Table 3, one can say that no clear trend can be seen with the pore diameter can be seen. This observation agrees with previous studies [24]. Indeed, according to these works, the cell size distribution should have no influence. Two aspects could explain the low equivalent conductivity of foam number $3\left(D_{\text {cell }}=\right.$ $5 \mathrm{~mm}$ ). On the one hand, its porosity is higher. On the other hand, the internal porosity due to the fabrication process (Section 3.1 and Fig. 7 c)) becomes very high relative to the size of the struts. This reduces the matter present in the bulk and thus reduces the equivalent conductivity of the solid matrix.

Concerning the influence of the geometry, we notice that strut organization and orientation have a significant influence. The measured optical thicknesses of foam number $1\left(D_{\text {cell }}=10 \mathrm{~mm}\right.$ tetra) and of foam number $4\left(D_{\text {cell }}=10 \mathrm{~mm}\right.$ cubic) are different because the organization of the solid matrices are different for these two samples. 
Only the sample thickness is different between foams number 4 (cubic $e=20 \mathrm{~mm}$ ) and 5 (cubic $e=15 \mathrm{~mm}$ ). Thus, in Fig. 12, we note a logical decrease of optical thickness, proportional to the decrease of the thickness of the samples. We find an extinction coefficient equal to $0.16 \mathrm{~mm}^{-1}$ for foam number 4 and equal to $0.14 \mathrm{~mm}^{-1}$ for foam number 5 . The values obtained are very similar and the difference can easily be explained by a slight difference of geometry due to the manufacturing process and the uncertainty due to a characterization at high temperature. The estimated equivalent radiative property seems to give a good description of the effective radiative transfer through the homogeneous media. Moreover, this confirms that the width of the investigated foams appears sufficient to consider the samples as an elementary volume representative of radiation transfers.

Fig. 9 shows the influence of the characterization temperature $T_{\infty}$ on the thermograms. A decrease of $300 \mathrm{~K}$ has a strong influence on the speed of the heat transfers. However, in Table 3 we see that this has no consequences for the estimated optical thickness. The radiative parameter is not influenced by the temperature, nor is the equivalent conductivity $\lambda_{\text {foam }}$ of the foam. A flash method experiment was applied on massive SiSiC discs. This additional thermal study revealed only a low variation of the thermal conductivity in this range of temperature. Moreover, an optical study of the ceramic showed that emissivity does not vary greatly in the range of wavelength where most of the radiative transfer takes place. Thus, Fig. 13 shows that the "radiative" conductivity $\lambda_{\text {rad }}$ is simply proportional to the cubic temperature of characterization $T_{\infty}^{3}$. Moreover, if we extrapolate the equivalent total conductivity at $T_{\infty}^{3}=0 \mathrm{~K}$ (i.e., only conduction), the result $\left(\lambda_{\text {tot }}(T=0)=\right.$ $\left.0.48 \mathrm{~W} \mathrm{~m}^{-1} \mathrm{~K}^{-1}\right)$ is very close to the equivalent conductivity estimated with the two-parameter model $\left(\bar{\lambda}_{\text {foam }} \approx 0.54 \mathrm{~W} \mathrm{~m}^{-1} \mathrm{~K}^{-1}\right)$. This result shows the ability of the present coupled model to separately characterize the influence of conduction and radiation transferts.

When comparing the equivalent conductivity (that occuring only by conduction) of structured to random foams in Fig. 11, we can see that the latter are less conductive. This can be explained by two effects. On the one hand, the strut material is different between $\mathrm{SiSiC}$ and $\mathrm{SiC}$. SiSiC is less conductive than the SiC. On the other hand, the struts of the random foams are smaller to the struts of the structured one. Hence, the closed porosity has a stronger impact on reducing the conduction heat within the solid matrix. This observation is in agreement with the findings of Coquard et al. [47] who demonstrated that conductivity is not only influenced by porosity but also by strut geometry.

The estimated equivalent conductivity $\lambda_{\text {foam }}$ is not dependent on the number of pores per inch. In Fig. 11, it can be seen that, for foams number $9(\mathrm{PPI}=40)$ and $10(\mathrm{PPI}=50)$ there is a high uncertainty on the thermal diffusivity (about $20 \%$ ) due to the correlation between the thermal and radiative parameters. When the equivalent optical thickness of the porous medium becomes too large, it gets difficult to separate the two transfer modes properly as shown above (see Section 2.2.5). In this case, we noticed an increase in the uncertainty associated with each of the estimated parameters. This is related to the fact that radiation transfer becomes similar to conduction transfer for high optical thicknesses (approximation of the diffusion resembles Rosseland's approximation).

The total equivalent conductivity $\lambda_{\text {tot }}$, estimated with a purely conductive model, does not match most of the experimental curves perfectly. However, good agreement was found for foam number 2 , which have a high $\lambda_{\text {foam }} / \lambda_{\text {tot }}(\%)$ ratio, and a very good agreement was found for foams number 8,9 and 10 , which have a high optical thicknesses (PPI $=(30,40,50))$ and where approximation of the diffusion is valid as explained immediately above. More generally, a purely conductive model describes both conductive and radiative heat transfers well for optical thicknesses above six. In such cases, the radiative transfer is thus comparable to a diffusive transfer.

For all cases, we noticed a better accuracy of the optical thickness than the equivalent conductivity $\lambda_{\text {foam }}$ characterized during the inversion process, due to the preponderance of the radiative transfer. Thus, the sensitivity associated with the first of these parameters is higher and allows a lower uncertainty according to Eq. (34). For example, for foam number 7 (PPI $=15$ ), the uncertainty due to the experimental noise introduces uncertainties of $4 \%$ for equivalent diffusivity (conduction only) and $0.65 \%$ for optical thickness.

\section{Conclusion}

The study of conduction and radiation occuring in heterogeneous media can be very complicated because of the coupling of the transfer modes. The main difficulty lies in the capacity to separate each heat transfer mode and to quantify their contribution. Usually, a single parameter is identified describing both conduction and radiation.

In order to get past this limitation, we developed a method based on a modified flash method experiment. The sample was placed between two soleplates and a constant heat flux was applied on the front face over a short time. The temporal evolution of the space-average temperature on the rear face was measured with an infrared camera. An inversion algorithm made it possible to identify both equivalent thermal conductivity and effective optical thickness. The procedure required a direct modeling of the problem. Hence, we developed a 2-D axisymetric geometry, taking into account all three layers. The model describes the transient heat transfer of a system including one semitransparent and two opaque media across a wide range of thermal and radiative properties. The Radiative Transfer Equation was solved with an optimized Monte Carlo method (Optimized Emission Reciprocity Method) while the Energy Balance Equation was computed with a Finite Volume Method. In order to solve the radiative transfer accurately, an Optimized Emission Reciprocity Method was used.

Two parameters, namely equivalent thermal conductivity $\lambda_{\text {foam }}$ and effective optical thickness $\tau$, were identified for several SiC and SiSiC foams. Moreover, we studied different kinds of ceramic, different cell sizes and different organizations of the solid matrix at temperatures up to $800{ }^{\circ} \mathrm{C}$ under vacuum. The link between structural properties and equivalent thermal properties of foams was investigated. Results showed that the equivalent thermal conductivity was strongly correlated with the strut material and with the internal porosity. Optical thickness depended on cell size and the shape of the cells. These results are in good agreement with observations made in previous studies. Thus, the proposed procedure appears to offer a notable improvement for research requiring an accurate and fast modeling of coupled transfers in complex porous geometries and by the ability to characterize the influence of each of the two heat transfer modes in a single experiment.

\section{Declaration of Competing Interest}

None.

\section{CRediT authorship contribution statement}

Morgan Sans: Conceptualization, Data curation, Formal analysis, Writing - original draft, Writing - review \& editing. Vincent Schick: Data curation, Formal analysis, Writing - original draft, Writing - review \& editing, Conceptualization. Gilles Parent: Conceptualization, Data curation, Formal analysis, Writing - original 
draft, Writing - review \& editing. Olivier Farges: Data curation, Formal analysis, Writing - original draft, Writing - review \& editing, Conceptualization.

\section{References}

[1] A. Sommers, Q. Wang, X. Han, C. T'Joen, Y. Park, A. Jacobi, Ceramics and ceramic matrix composites for heat exchangers in advanced thermal systems a review, Appl. Therm. Eng. 30 (11-12) (2010) 1277-1291, doi:10.1016/ j.applthermaleng.2010.02.018.

[2] J.R. Howell, M.J. Hall, J.L. Ellzey, Combustion of hydrocarbon fuels within porous inert media, Prog. Energy Combust. Sci. 22 (2) (1996) 121-145

[3] J. Randrianalisoa, Y. Bréchet, D. Baillis, Materials selection for optimal design of a porous radiant burner for environmentally driven requirements, Adv. Eng. Mater. (2009), doi:10.1002/adem.200900089.

[4] R. Coquard, D. Baillis, Modeling of heat transfer in low-density EPS foams, J. Heat Transf. 128 (6) (2006) 538, doi:10.1115/1.2188464.

[5] E. Placido, M. Arduini-Schuster, J. Kuhn, Thermal properties predictive model for insulating foams, Infrared Phys. Technol. 46 (3) (2005) 219-231, doi:10. 1016/j.infrared.2004.04.001.

[6] S. Ishiyama, S. Maruyama, Compact heat exchanger made of ceramics having corrosion resistance at high temperature, 2011, US Patent 7,981,168.

[7] E. Velasco Gómez, F. Rey Martínez, F. Varela Diez, M. Molina Leyva, R. Herrero Martín, Description and experimental results of a semi-indirect ceramic evaporative cooler, Int. J. Refrig. 28 (5) (2005) 654-662, doi:10.1016/j.jirefrig.2005. 01.004.

[8] R. Smyth, A proposal for the use of a very high temperature ceramic heat exchanger in gas turbine power production, in: Energy Conversion Engineering Conference, 1997. IECEC-97., Proceedings of the 32nd Intersociety, 3, IEEE, 1997, pp. 1696-1701.

[9] Z. Wu, C. Caliot, G. Flamant, Z. Wang, Coupled radiation and flow modeling in ceramic foam volumetric solar air receivers, Solar Energy 85 (9) (2011) 2374 2385, doi:10.1016/j.solener.2011.06.030.

[10] T. Fend, B. Hoffschmidt, R. Pitz-Paal, O. Reutter, P. Rietbrock, Porous materials as open volumetric solar receivers: experimental determination of thermophysical and heat transfer properties, Energy 29 (5-6) (2004) 823-833. doi:10.1016/S0360-5442(03)00188-9.

[11] J. Lévêque, D. Rouzineau, M. Prévost, M. Meyer, Hydrodynamic and mass transfer efficiency of ceramic foam packing applied to distillation, Chem. Eng. Sci. 64 (11) (2009) 2607-2616, doi:10.1016/j.ces.2009.02.010

[12] T. Kim, Micro methanol reformer combined with a catalytic combustor for a PEM fuel cell, Int. J. Hydrog. Energy 34 (16) (2009) 6790-6798, doi:10.1016/j. ijhydene.2009.06.024.

[13] E. Jahanshahi Javaran, S. Gandjalikhan Nassab, S. Jafari, Thermal analysis of a 2-D heat recovery system using porous media including lattice Boltzmann simulation of fluid flow, Int. J. Therm. Sci. 49 (6) (2010) 1031-1041, doi:10.1016/j. ijthermalsci.2009.12.004.

[14] J.M. Chavez, C. Chaza, Testing of a porous ceramic absorber for a volumetric air receiver, Solar Energy Mater. 24 (1-4) (1991) 172-181.

[15] T. Fend, B. Hoffschmidt, R. Pitz-Paal, O. Reutter, P. Rietbrock, Porous materials as open volumetric solar receivers: experimental determination of thermophysical and heat transfer properties, Energy 29 (5-6) (2004) 823833.

[16] P. Ranut, On the effective thermal conductivity of aluminum metal foams: review and improvement of the available empirical and analytical models, Appl. Therm. Eng. 101 (2016) 496-524, doi:10.1016/j.applthermaleng.2015.09.094.

[17] M.N. Miller, Bounds for effective electrical, thermal, and magnetic properties of heterogeneous materials, J. Math. Phys. 10 (11) (1969) 1988-2004, doi:10. $1063 / 1.1664794$.

[18] Z. Hashin, S. Shtrikman, A variational approach to the theory of the effective magnetic permeability of multiphase materials, J. Appl. Phys. 33 (10) (1962) 3125-3131, doi:10.1063/1.1728579.

[19] R. Landauer, The electrical resistance of binary metallic mixtures, J. Appl. Phys. 23 (7) (1952) 779-784, doi:10.1063/1.1702301.

[20] J.W. Paek, B.H. Kang, S.Y. Kim, J.M. Hyun, Effective thermal conductivity and permeability of aluminum foam materials, Int. J. Thermophys. 21 (2) (2000) 453-464.

[21] C.T. Hsu, P. Cheng, K.W. Wong, Modified Zehner-Schlunder models for stagnant thermal conductivity of porous media, Int. J. Heat Mass Transf. 37 (17) (1994) $2751-2759$

[22] V. Calmidi, R. Mahajan, The effective thermal conductivity of high porosity fibrous metal foams, J. Heat Transf. 121 (2) (1999).

[23] A. Bhattacharya, V. Calmidi, R. Mahajan, Thermophysical properties of high porosity metal foams, Int. J. Heat Mass Transf. 45 (5) (2002) 1017 1031
[24] K. Boomsma, D. Poulikakos, On the effective thermal conductivity of a threedimensionally structured fluid-saturated metal foam, Int. J. Heat Mass Transf. 44 (4) (2001) 827-836

[25] R. Singh, H. Kasana, Computational aspects of effective thermal conductivity of highly porous metal foams, Appl. Therm. Eng. 24 (13) (2004) 1841-1849.

[26] R. Coquard, D. Baillis, Numerical investigation of conductive heat transfer in high-porosity foams, Acta Mater. 57 (18) (2009) 5466-5479, doi:10.1016/j. actamat.2009.07.044.

[27] S. Cunsolo, R. Coquard, D. Baillis, N. Bianco, Radiative properties modeling of open cell solid foam: review and new analytical law, Int. J. Therm. Sci. 104 (2016) 122-134, doi:10.1016/j.ijthermalsci.2015.12.017.

[28] B. Zeghondy, E. Iacona, J. Taine, Determination of the anisotropic radiative properties of a porous material by radiative distribution function identification (RDFI), Int. J. Heat Mass Transf. 49 (17-18) (2006) 2810-2819, doi:10.1016/j. ijheatmasstransfer.2006.02.034.

[29] M. Tancrez, J. Taine, Direct identification of absorption and scattering coefficients and phase function of a porous medium by a monte carlo technique Int. J. Heat Mass Transf. 47 (2) (2004) 373-383, doi:10.1016/S0017-9310(03) 00146-7.

[30] S. Guévelou, B. Rousseau, G. Domingues, J. Vicente, C. Caliot, Representative elementary volumes required to characterize the normal spectral emittance of silicon carbide foams used as volumetric solar absorbers, Int. J. Heat Mass Transf. 93 (2016) 118-129, doi:10.1016/j.ijheatmasstransfer.2015.09.069.

[31] M. Loretz, R. Coquard, D. Baillis, E. Maire, Metallic foams: radiative properties/comparison between different models, J. Quant. Spectrosc. Radiat. Transf 109 (1) (2008) 16-27.

[32] M.A.A. Mendes, V. Skibina, P. Talukdar, R. Wulf, U. Gross, D. Trimis, S. Ray, Experimental validation of simplified conduction-radiation models for evaluation of effective thermal conductivity of open-cell metal foams at high temperatures, Int. J. Heat Mass Transf. 78 (2014) 112-120.

[33] M.A. Mendes, P. Talukdar, S. Ray, D. Trimis, Detailed and simplified models for evaluation of effective thermal conductivity of open-cell porous foams at high temperatures in presence of thermal radiation, Int. J. Heat Mass Transf 68 (2014) 612-624

[34] D. Baillis, R. Coquard, J.H. Randrianalisoa, L.A. Dombrovsky, R. Viskanta, Thermal radiation properties of highly porous cellular foams, Spec. Top. Rev. Porous Media 4 (2) (2013).

[35] M. Lazard, S. André, D. Maillet, Diffusivity measurement of semi-transparent media: model of the coupled transient heat transfer and experiments on glass, silica glass and zinc selenide, Int. J. Heat Mass Transf. 47 (3) (2004) 477-487, doi:10.1016/j.ijheatmasstransfer.2003.07.003.

[36] V. Schick, B. Remy, A. Degiovanni, F. Demeurie, J. Meulemans, P. Lombard, Measurement of thermal conductivity of liquids at high temperature, J. Phys. 395 (2012) 012078, doi:10.1088/1742-6596/395/1/012078.

[37] M. Niezgoda, D. Rochais, F. Enguehard, P. Echegut, B. Rousseau, Modeling of time-resolved coupled radiative and conductive heat transfer in multilayer semitransparent materials up to very high temperatures, Appl. Phys. Lett. 99 (22) (2011) 224101

[38] R. Coquard, D. Rochais, D. Baillis, Experimental investigations of the coupled conductive and radiative heat transfer in metallic/ceramic foams, Int. J. Heat Mass Transf. 52 (21-22) (2009) 4907-4918, doi:10.1016/j.ijheatmasstransfer 2009.05.015.

[39] M.F. Modest, Radiative heat transfer, Academic press, 2013.

[40] B. Le Corre, A. Collin, L. Soudre-Bau, Y. Meshaka, G. Jeandel, Glass sagging simulation with improved calculation of radiative heat transfer by the optimized reciprocity monte carlo method, Int. J. Heat Mass Transf. 70 (2014) 215-223.

[41] L. Tessé, F. Dupoirieux, B. Zamuner, J. Taine, Radiative transfer in real gases using reciprocal and forward monte carlo methods and a correlated-k approach, Int. J. Heat Mass Transf. 45 (13) (2002) 2797-2814

[42] Y. Zhang, O. Gicquel, J. Taine, Optimized emission-based reciprocity monte carlo method to speed up computation in complex systems, Int. J. Heat Mass Transf. 55 (25-26) (2012) 8172-8177.

[43] J.J. Moré, The Levenberg-Marquardt algorithm: implementation and theory, in: Numerical Analysis, Springer, 1978, pp. 105-116.

[44] H. Bal, Y. Jannot, N. Quenette, A. Chenu, S. Gaye, Water content dependence of the porosity, density and thermal capacity of laterite based bricks with millet waste additive, Constr. Build. Mater. 31 (2012) 144-150.

[45] M. Lazard, S. Andre, D. Maillet, Modèle semi-analytique de couplage conduction rayonnement dans un milieu semi-transparent absorbant, émettant et diffusant de façon non isotrope., Rayonnement et Milieux Semi-Transparents. Journée, 2000

[46] V. Schick, B. Remy, A. Degiovanni, F. Demeurie, J. Meulemans, P. Lombard, Measurement of thermal conductivity of liquids at high temperature, in: Journal of Physics: Conference Series, 395, IOP Publishing, 2012, p. 012078.

[47] R. Coquard, M. Loretz, D. Baillis, Conductive heat transfer in metallic/ceramic open-cell foams, Adv. Eng. Mater. 10 (4) (2008) 323-337. 